\title{
Motorized capsule for shadow-free OCT imaging and synchronous beam control
}

\author{
Antonio López-Marín, ${ }^{1}$ (1) Geert Springeling, ${ }^{1}$ Robert Beurskens, ${ }^{1}$ Heleen van Beusekom, ${ }^{1}$ \\ Antonius F. W. van der Steen, ${ }^{1,2,3}$ ArJun D. Koch, ${ }^{1}$ Brett E. Bouma, ${ }^{4,5}$ Robert Huber, ${ }^{6}$ (i) \\ GiJs van Soest, ${ }^{1}$ and TIANSHI WANG $^{1, *}$ \\ ${ }^{1}$ Erasmus University Medical Center, P. O. Box 2040, Rotterdam 3000 CA, The Netherlands \\ ${ }^{2}$ Shenzhen Institutes of Advanced Technology, Chinese Academy of Sciences, Shenzhen, 518005, China \\ ${ }^{3}$ Department of Imaging Science and Technology, Delft University of Technology, Delft, 2600 AA, The Netherlands \\ ${ }^{4}$ Wellman Center for Photomedicine, Harvard Medical School and Massachusetts G.H., 40 Blossom Street, Boston, Massachusetts 02114, USA \\ ${ }^{5}$ Harvard-MIT Program in Health Sciences and Technology, Cambridge, Massachusetts 02139, USA \\ ${ }^{6}$ Institut für Biomedizinische Optik, Universität zu Lübeck, Lübeck, 23562, Germany \\ *Corresponding author: t.wang.1@erasmusmc.nl
}

Received 19 April 2019; revised 17 June 2019; accepted 25 June 2019; posted 26 June 2019 (Doc. ID 365429); published 18 July 2019

We demonstrate a tethered motorized capsule for unobstructed optical coherence tomography (OCT) imaging of the esophagus. By using a distal reflector design, we avoided the common shadow artifact induced by the motor wires. A synchronous driving technique features three types of beam-scanning modes of the capsule, i.e., circumferential beam scanning, localized beam scanning, and accurate beam positioning. We characterized these three modes and carried out ex vivo imaging experiments using the capsule. The results show that the capsule can potentially be a useful tool for diagnostic OCT imaging and OCT-guided biopsy and therapy of the esophagus. (c) 2019 Optical Society of America

https://doi.org/10.1364/OL.44.003641

Provided under the terms of the OSA Open Access Publishing Agreement

Optical coherence tomography (OCT), as an optical imaging technology, has been widely used for medical diagnosis and industrial nondestructive testing [1-3]. Making use of lowcoherence light, OCT captures the morphological structure of the tissue with a micrometer order resolution and with millimeter order penetration depth. To acquire diagnostic OCT images of the internal organs, an imaging catheter is used to shine the imaging beam upon the lumen of the organ and collect the back-reflected light for image reconstruction. Typically, a motorized rotary joint rotates a long fiber probe inside the catheter to achieve circumferential beam scanning of the entire lumen [2]. A volumetric dataset of the lumen can be acquired by pulling back the probe during beam scanning.

In 2008, Seibel et al. demonstrated a new endoscope design for diagnostic imaging in the esophagus, which involves swallowing a tethered capsule that can acquire diagnostic images as it travels through the digestive tract [4]. In 2013, Gora et al. demonstrated a tethered capsule for OCT imaging in the esophagus [5]. The ability to perform laser coagulation to mark suspicious areas for biopsy has also been demonstrated in esophageal OCT imaging [6]. Lately, several novel capsule designs have been proposed, including motorized capsules that integrate a micromotor into the capsule to perform beam scanning [7-9]. Without rotating the long fiber probe, distal beam scanning can achieve better stability and higher scanning speed. However, the motor wires block the light during beam scanning and induce one or more shadow artifacts in the OCT image [7-10]. These may potentially affect the diagnosis and laser marking of the esophagus, especially when the area of interest coincidentally lies in the wire shadow. A micromotor using glass components was developed to eliminate shadows but either constrains the optical design by the mechanical structure of the motor or limits the maximum rotational speed [11-13]. Recently, Liang et al. demonstrated beam positioning for laser marking using a stepper motor in the capsule (with a wire shadow), which, however, can hold the beam only at specific positions [14].

In this study, we demonstrate a motorized tethered capsule, in which the OCT light beam goes around the motor to avoid the shadow artifact. Beyond the unobstructed circumferential beam scanning achieved, a synchronous driving technique features the capsule's two advanced beam-scanning modes, i.e., accurate beam positioning and localized beam scanning.

The schematic diagram of the motorized capsule is shown in Fig. 1. Unlike the conventional design that puts the micromotor and the fiber probe facing each other, in our capsule,

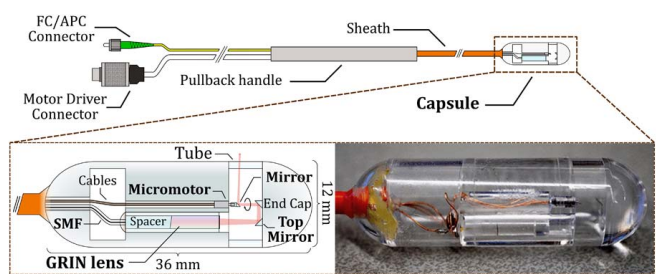

Fig. 1. Schematic of the tethered capsule and photo of the capsule. 
a micromotor (Kinetron, Netherlands) and a customized gradient refractive index (GRIN) lens probe (Go!Foton, United States) were placed side by side facing the distal cap. Two $1.0 \mathrm{~mm}$ mirrors (Edmund, 86-621, United States) were attached to the distal cap of the capsule to deflect the OCT light twice, thus turning the light beam around. The deflected light beam finally falls upon the third $0.7 \mathrm{~mm}$ mirror (Edmund, 66-771, United States) that is attached on the motor shaft. By using such a design, the motor wires will not cross the optical path, and the shadow artifacts in the OCT image are thus avoided. Compared to conventional designs, this configuration makes use of two additional mirrors, which increases the length of the optical path in the capsule. To ensure that the optical beam can still focus on the esophagus outside the capsule surface, a large GRIN lens $(13 \mathrm{~mm}$ rigid length and $2.8 \mathrm{~mm}$ diameter) with a long focal length of approximately $20 \mathrm{~mm}$ was used. It is worth mentioning that the GRIN lens can still be easily fit into the capsule, as shown in Fig. 1. Besides the increase in optical path length, the two mirrors also induce additional power loss. The reflectivity of each aluminumcoated mirror was measured to be $>93.6 \%$ near $1310 \mathrm{~nm}$, adding a two-way total power loss of $<23.2 \%(-2.3 \mathrm{~dB}$ loss in sensitivity compared to an overall sensitivity of $96 \mathrm{~dB}$ ).

The micromotor developed for this capsule is a two-phase four-pole synchronous motor, which consists of a permanent magnet rotor and a stator copper coil (0.3 $\Omega$ resistance). The motor is $2.0 \mathrm{~mm}$ long and has a $1.0 \mathrm{~mm}$ diameter. The details of the coil design and thermal test can be found in our previous study [15]. The micromotor is controlled in an open-loop configuration by driving it with a pair of sinusoidal currents, and the rotational speed (in rev/s) of the motor is equal to the frequency of the current signal. Furthermore, the angular position of the magnetic rotor, and thus the angle of the light beam, follows the direction of the magnetic field generated by the current in the coils [15]. As shown in Fig. 2(a), the direction of the magnetic field is set by the phase of the current signal from the two channels. The finite element simulation of the magnetic field (ANSYS Maxwell, 16.0, U.S.) in Fig. 2(b)
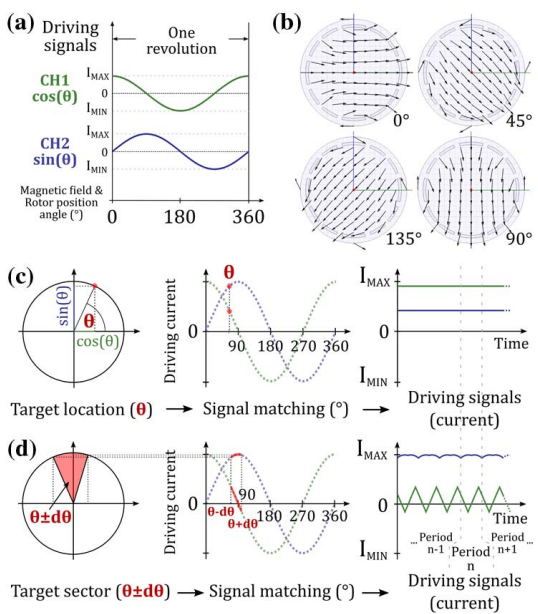

Fig. 2. (a) Principle of the synchronous control; (b) magnetic field simulation of the micromotor setting the phase of the driving current at $0^{\circ}, 45^{\circ}, 90^{\circ}$, and $135^{\circ}$; (c), (d) schematic diagram of control modes from ideal angular position to current signal: (c) beam positioning control and (d) localized beam scanning control. shows that the direction of the magnetic field changes with the phase value of the current signals.

We designed three different working modes of the capsule based on the synchronous control principle: circumferential beam-scanning mode, beam-positioning mode, and localized beam-scanning mode. The working principles of the latter two modes are presented in Figs. 2(c) and 2(d), showing how the motor-driving signals were created based on the target location and target-scanning sector. Positioning control feeds direct currents of magnitude $I \sin (\theta)$ and $I \cos (\theta)$ to the two channels, thus creating a constant magnetic field to "hold" the rotator at one specific angular position $\theta$ [Fig. 2(c)]. Localized scanning mode is developed based on the positioning mode, where the current signal varies near the center position $\theta$ with a pre-defined angular range of $\pm d \theta$ [Fig. 2(d)]. To that end, we implemented a virtual instrument (LabVIEW, United States) to control the light beam in real time.

The tethered capsule was made by poly(methyl methacrylate) and prepared in three parts separately using computer numerical control (CNC) machining: the distal part consisting of the end cap and the two additional mirrors; the proximal part consisting of the body of the plastic capsule, the micromotor and the GRIN lens probe; and the tail part consisting of the protective tube, the single-mode fiber, and the motor cables. After all of these parts are assembled, the final dimensions of the capsule are as follows: length of $36 \mathrm{~mm}$, diameter of $12 \mathrm{~mm}$, and a tail length of $1.6 \mathrm{~m}$. The micrometer-order accuracy of the CNC milling process ensures accurate positioning and alignment of the optics.

The $1.0 \mathrm{~mm}$ thick outer tube of the capsule acts as a convex cylindrical lens and induces astigmatism to the beam. The light beam is defocused along the azimuthal axis that is perpendicular to the light beam and the capsule axis (longitudinal axis). Using a beam profiler (Thorlabs, BP-209, United States), we characterized the beam as: $0.9 \mathrm{~mm}$ focal distance $(1.0 \mathrm{~mm}$ target focal distance outside the capsule) with $32.5 \mu \mathrm{m}$ spot size along the longitudinal axis and $1.6 \mathrm{~mm}$ focal distance with $34.0 \mu \mathrm{m}$ spot size along the azimuthal axis; beam waist locations measured relative to the capsule outer wall. Though the beam is slightly defocused due to astigmatism, the Gaussian beam spot size is still 34-39 $\mu \mathrm{m}$ within the imaging depth.

We characterized the rotating speed, accuracy of beam positioning, and localized beam-scanning range. To measure the rotational speed, we used a homemade setup as described previously [15]. To characterize beam positioning and localized beam scanning, we put the capsule in the center of a cylindrical tube that had 360 markers covering $0-360^{\circ}$. Connecting the capsule to a $633 \mathrm{~nm}$ laser, the light spot can be found on the tube, and the angular position of the spot can be measured with an accuracy of $1^{\circ}$. We measured the position of the spot by changing the phase of the driving current from $0^{\circ}-360^{\circ}$ with $1^{\circ}$ increments. The localized scanning range was set to $\pm 15^{\circ}$ with a scanning rate of $50 \mathrm{~Hz}$. The current of the motor was set to $500 \mathrm{~mA}$ (effective value) for all measurements and imaging experiments.

The rotating speed was characterized at 500 revolutions/s, and the maximum speed error was found to be $3.55 \%$ with a standard deviation of $1.98 \%$. Though the speed ripple was small, non-uniform rotation distortion (NURD) would still affect the circumferential beam scanning and induce some wobbling to the OCT images. 


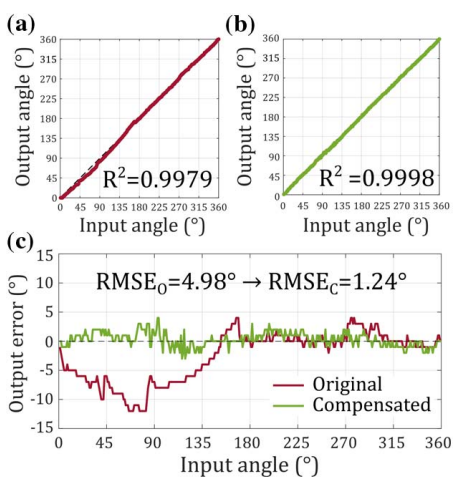

Fig. 3. (a) Original angular positioning profile; (b) compensated angular positioning profile; (c) angle error from (a) and (b); $\mathrm{RMSE}_{\mathrm{O}, \mathrm{C}}$ : root mean squared error for original/compensated control cases.

Experimental results of the beam positioning are shown in Fig. 3. It can be seen that the output angular position of the light beam is synchronous to the input phase of the current. In the range of $0^{\circ}-135^{\circ}$, the error between input angle and output angle is larger than it is for the rest of the range, which may be induced by a speck of dust or a manufacturing irregularity. To compensate for the positioning error, for each specific output angular position $\theta$, we looked for the corresponding input angle in Fig. 3 and used the retrieved value as the corrected input current signals. After applying the compensation, the output angle error was significantly depressed, and the root mean square error (RMSE) was reduced from $4.98^{\circ}$ to $1.24^{\circ}$. The measured output angle is shown in Figs. 3(a) and 3(b), and the positioning error is plotted in Fig. 3(c). A video was recorded to demonstrate real-time beam-positioning control (Visualization 1).

As shown in Fig. 4, the dashed line indicates the center angular position of localized beam scanning, and two solid lines indicate the upper and lower bounds of the scan range separately. Similar to the beam positioning measurement, a relatively large error of the center position was found from $0^{\circ}$ to $135^{\circ}$. However, the error of the scanning range was found to be $<3^{\circ}$ for all localized scanning measurements. Compensation similar to the positioning control was applied, mainly to improve the accuracy of the center position. The RMSE was reduced from $4.9^{\circ}$ to $2.0^{\circ}$ after applying compensation; the error

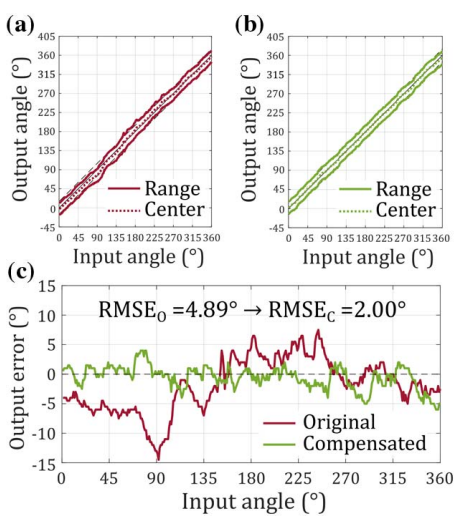

Fig. 4. (a) Original localized beam-scanning profile; (b) compensated localized beam-scanning profile; (c) angle error from (a) and (b). of scan range was maintained at $<3^{\circ}$. A video was recorded to demonstrate real-time control of the localized beam scanning (Visualization 2).

Ex vivo imaging experiments were carried out in a swine esophagus in accordance with a protocol sanctioned by the local ethics committee. The capsule was connected to an OCT imaging system that was built based on a $1.5 \mathrm{MHz}$ Fourier domain mode-locked (FDML) laser. The FDML-OCT system offers an imaging depth of $4.0 \mathrm{~mm}$, axial resolution of $12 \mu \mathrm{m}$, and sensitivity of $96 \mathrm{~dB}$ [16]. The frequency of the driving current was set to $500 \mathrm{~Hz}$ to achieve a frame rate of 500 frames/s and a sufficient axial scan (A-scan) density of 3112 lines per frame. To acquire a volumetric dataset, the capsule was pulled back from the lower towards the upper part of the esophagus at a speed of $15 \mathrm{~mm} / \mathrm{s}$. Due to the $\mathrm{MHz}$ sweep rate of the FDML laser, a high frame rate, high A-scan density, and fast pullback could be achieved at the same time.

Figure 5 and a video recording (Visualization 3) show the imaging results of the esophagus. The lumen of the entire esophagus can be seen in the cross-sectional OCT image (Fig. 5) without any shadow artifact, proving that the capsule can provide unobstructed OCT imaging. The three-dimensional reconstruction in Fig. 5 also shows the entire esophagus without any shadow artifact. The layered structure of the esophagus, i.e., the epithelium, lamina propria, muscularis mucosae, submucosae, and muscularis propria, are identifiable in the zoomed area, as shown in Fig. 5.

To validate the localized imaging enabled by localized beam scanning, we first acquired circumferential images at 500 frames/s. Then we switched the scanning mode to localized beam scanning and acquired localized images every $30^{\circ}$. The frame rate was set to 50 frames/s according to the $50 \mathrm{~Hz}$ scanning rate. Figure 6 compares the images acquired at the circumferential beam-scanning mode (center) and the localized beam-scanning mode (outer ring). The dynamic ranges for both imaging modes were optimized separately. The structural features in each localized image are the same as those in the corresponding part of the circumferential image, which proves the accuracy of localized beam scanning. More structural features (star mark) can be seen in the localized image because every 12 A-scans were averaged to further improve sensitivity. Though NURD-like artifacts induced by the acceleration and deceleration of the micromotor can be seen in the margins of the localized images, the image features can still be interpreted. Last, we combined all the localized images into one rectangular image [Fig. 6(c)] and compared that with the original circumferential image [Fig. 6(b)] within the same dynamic range. Though NURD artifacts can still be seen in the combined
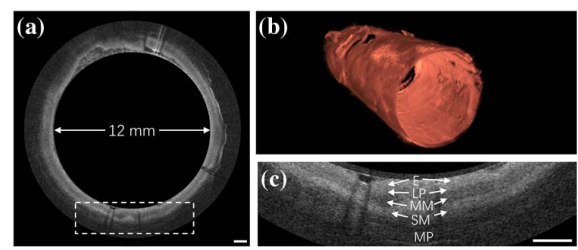

Fig. 5. (a) Representative cross-sectional image of swine esophagus (inner wall of the tube is cropped out); (b) 3D reconstruction along a $4.5 \mathrm{~cm}$ pull-back distance; (c) zoomed-in area of the dotted box in (b). E, epithelium; LP, lamina propria; MM, muscularis mucosae; SM, submucosae; MP, muscularis propria. Scale bar is $1 \mathrm{~mm}$. 


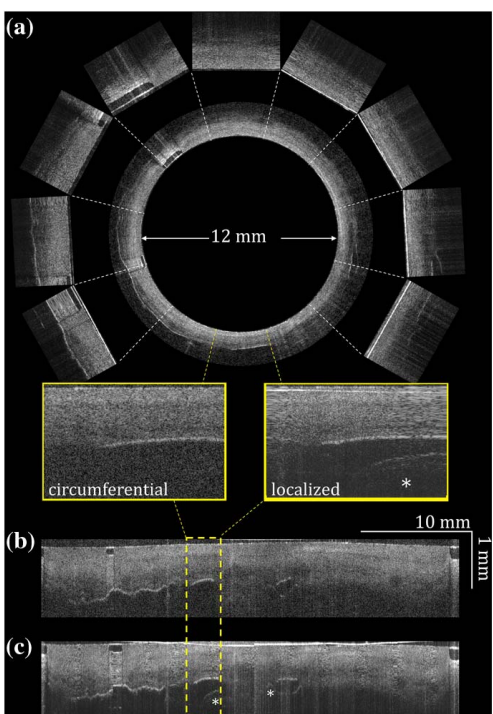

Fig. 6. (a) Comparison between the OCT image of the esophagus acquired at circumferential beam-scanning mode (center) and at localized beam-scanning mode (outer ring). (b) Rectangular images of esophagus acquired at circumferential beam-scanning mode. (c) Image reconstructed by combining all images acquired at localized beamscanning mode; (b) and (c) have the same dynamic range.

image, Fig. 6(c) shows more features (star marks) than Fig. 6(b) and also has lower noise. A video was recorded to show the accurate localized imaging for every $30^{\circ}$ (Visualization 4). Another video was created to demonstrate how the beam was held upon a $0.9 \mathrm{~mm}$ diameter tube utilizing the three beamscanning modes (Visualization 5).

In summary, we demonstrate a tethered motorized capsule that can provide shadow-free OCT images of the esophagus. A synchronous driving technique features the capsule's three beam-scanning modes, i.e., unobstructed circumferential scanning mode, beam-positioning mode, and localized beamscanning mode. The circumferential mode can be used for fast screening of the entire esophagus. The localized beam-scanning mode allows us to zoom in to suspicious areas locally and see the features better at any angular position. Finally, the beampositioning mode has an accuracy of $1.24^{\circ}$, which could be used to place a laser mark precisely. In our study, the optics design requires only two additional mirrors to get rid of the shadow artifact and does not require any re-engineering of the motor itself. We believe such a design can be adopted by those catheter or capsule designs that have motor wire obstruction.

Future prototypes will feature a reduced length of the capsule of $25 \mathrm{~mm}$, by further reducing extraneous space, which also allows the use of a GRIN lens with shorter focal length. Losses in the fiber probe will be negligible for when bending radii larger than $25 \mathrm{~mm}$. The artifact induced by the acceleration and deceleration of the motor is still an issue for localized imaging, which can potentially be compensated for by re-sampling the A-scans according to the momentary speed of the motor. The capsule can accommodate a micromotor several millimeters larger. With such dimensions, the manufacturing difficulties and thus the cost of the motor can be significantly reduced. Beyond the imaging and laser marking in the esophagus, localized beam scanning may also enable localized laser-based therapy and real-time monitoring. In the next step, we will carry out in vivo tests of the capsule, develop motors with 3-4 mm diameter, and investigate localized therapy and monitoring using the capsule.

Funding. Nederlandse Organisatie voor Wetenschappelijk Onderzoek (NWO) (Veni-15940, ZonMW 104003006); Deutsche Forschungsgemeinschaft (DFG) (DFG- EXC 2167); H2020 European Research Council (ERC) (ERC CoG_ ENCOMOLE-2i, 646669).

Acknowledgment. The authors acknowledge the late Dr. Charles Lancée for inventing the micromotor and inspiring this work. The authors acknowledge Mr. Tom Pfeiffer and Dr. Wolfgang Wieser for their contributions to the development of the OCT system. The authors acknowledge Mr. John Meijer for his contribution to the motor manufacturing.

\section{REFERENCES}

1. D. Huang, E. A. Swanson, C. P. Lin, J. S. Schuman, W. G. Stinson, W. Chang, M. R. Hee, T. Flotte, K. Gregory, C. A. Puliafito, and J. G. Fujimoto, Science 254, 1178 (1991).

2. G. J. Tearney, M. E. Brezinski, B. E. Bouma, S. A. Boppart, C. Pitris, J. F. Southern, and J. G. Fujimoto, Science 276, 2037 (1997).

3. M. R. Hee, J. A. Izatt, E. A. Swanson, D. Huang, J. S. Schuman, C. P. Lin, C. A. Puliafito, and J. G. Fujimoto, Arch Ophthalmol. 113, 325 (1995).

4. E. J. Seibel, R. E. Carroll, J. A. Dominitz, R. S. Johnston, C. D. Melville, C. M. Lee, S. M. Seitz, and M. B. Kimmey, IEEE Trans. Biomed. Eng. 55, 1032 (2008).

5. M. J. Gora, J. S. Sauk, R. W. Carruth, K. A. Gallagher, M. J. Suter, N. S. Nishioka, L. E. Kava, M. Rosenberg, B. E. Bouma, and G. J. Tearney, Nat. Med. 19, 238 (2013).

6. M. J. Suter, M. J. Gora, G. Y. Lauwers, T. Arnason, J. Sauk, K. A. Gallagher, L. Kava, K. M. Tan, A. R. Soomro, T. P. Gallagher, J. A. Gardecki, B. E. Bouma, M. Rosenberg, N. S. Nishioka, and G. J. Tearney, Gastrointest. Endosc. 79, 886 (2014).

7. K. Liang, Z. Wang, O. O. Ahsen, H. C. Lee, B. M. Potsaid, V. Jayaraman, A. Cable, H. Mashimo, X. Li, and J. G. Fujimoto, Optica 5, 36 (2018).

8. K. Liang, G. Traverso, H. C. Lee, O. O. Ahsen, Z. Wang, B. Potsaid, M. Giacomelli, V. Jayaraman, R. Barman, A. Cable, H. Mashimo, R. Langer, and J. G. Fujimoto, Biomed. Opt. Express 6, 1146 (2015).

9. K. Li, W. Liang, J. Mavadia-Shukla, H. C. Park, D. Li, W. Yuan, S. Wan, and X. Li, J. Biophoton. 12, e201800205 (2019).

10. T. Wang, W. Wieser, G. Springeling, R. Beurskens, C. T. Lancee, T. Pfeiffer, A. F. van der Steen, R. Huber, and G. van Soest, Opt. Lett. 38, 1715 (2013).

11. T. Chen, N. Zhang, T. Huo, C. Wang, J. G. Zheng, T. Zhou, and P. Xue, J. Biomed. Opt. 18, 86011 (2013).

12. https://www.ad-na.com/en/product/motor-unit/oct.html.

13. S. Chang, E. Murdock, Y. Mao, C. Flueraru, and J. Disano, Opt. Lett. 36, 4392 (2011).

14. C.-P. Liang, J. Dong, T. Ford, R. Reddy, H. Hosseiny, H. Farrokhi, M. Beatty, K. Singh, H. Osman, B. Vuong, G. Baldwin, C. Grant, S. Giddings, M. J. Gora, M. Rosenberg, N. Nishioka, and G. Tearney, Biomed. Opt. Express 10, 1207 (2019).

15. T. Wang, C. Lancée, R. Beurskens, J. Meijer, B. Knapen, A. F. W. van der Steen, and G. van Soest, Sens. Actuators A, Phys. 218, 60 (2014).

16. T. Wang, T. Pfeiffer, M. Wu, W. Wieser, G. Amenta, W. Draxinger, A. F. W. van der Steen, R. Huber, and G. V. Soest, Opt. Lett. 42, $3466(2017)$ 